\title{
ESTILO DE VIDA E FATORES DE RISCO DE PACIENTES COM PRIMEIRO EPISÓDIO DE INFARTO AGUDO DO MIOCÁRDIO*
}

Roberta Cunha Rodrigues Colombo ** Olga Maimoni Aguillar ***

COLOMBO, R.C.R.; AGUILLAR, O.M. Estilo de vida e fatores de risco de pacientes com primeiro episódio de infarto agudo do miocárdio. Rev.latino-am.enfermagem, Ribeirão Preto, v. 5, n. 2, p. 69-82, abril 1997.

A prevenção do infarto agudo do miocárdio (IAM) relaciona-se com a identificação e controle dos fatores de risco (FR) presentes no estilo de vida dos indivíduos. Tal estudo propõe-se a conhecer o perfil dos pacientes com um primeiro episódio de IAM, identificando os FR para essa doença. Foram estudados 78 pacientes, atendidos na Unidade Coronária de um Hospital Universitário. Os resultados mostraram que o grupo estudado possui, em seu estilo de vida, hábitos que propiciam a manutenção elou progressão de coronariopatia. Estes achados subsidiarão a elaboração de um programa educacional, com vistas à prevenção de reinfarto e promoção de saúde.

UNITERMOS: infarto do miocárdio, estilo de vida, fatores de risco

\section{INTRODUÇÃO}

As doenças cardiovasculares representam uma das maiores causas de mortalidade em todo o mundo. Antigamente essas doenças eram consideradas causa importante de óbito somente em países desenvolvidos. Hoje, mesmo nos países em desenvolvimento, com um maior controle das doenças infecto-parasitárias e conseqüente aumento da longevidade de suas populações, essa doença tem atingido um número cada vez maior de pessoas em uma faixa etária cada vez mais baixa (BANCO MUNDIAL, 1991).

Entre as doenças cardiovasculares, a de maior incidência é a doença arterial coronária (DAC) cujas principais manifestações clínicas são a angina pectoris, o infarto agudo do miocárdio (IAM) e a morte súbita (BATLOUNI, 1993).

O que poderia estar dificultando a prevenção desta doença em nossa população? Essa indagação passa a ter uma importância ainda maior, se levarmos em consideração que já há mais de três décadas foram estabelecidos os chamados fatores de risco (FR) associados ao desenvolvimento dessa doença, os quais deveriam conferir-lhe a possibilidade de uma prevenção efetiva, tanto em nível individual como coletivo.

O termo "fator de risco" surgiu pela primeira vez quando KANNEL et al. (1961) divulgaram os achados do Framingham Heart Study. Esse estudo pioneiro realizou seguimento de uma amostra populacional de aproximadamente 5000 indivíduos dos sexos masculino e feminino, residentes na cidade de Framingham, Massachusetts, objetivando identificar FR ao se comparar indivíduos que tinham desenvolvido DAC com aqueles que não tinham. Portanto "tudo que foi medido e mais tarde relacionado com uma maior incidência de doença foi denominado fator de risco, isto é, fator que desempenha um papel no desenvolvimento da doença" (MANCILHA-CARVALHO, 1992).

De acordo com CUNNINGHAM (1992) os FR podem ser classificados em modificáveis e não modificáveis. Os últimos incluem idade, sexo, raça e história familiar de doença aterosclerótica. Os FR modificáveis, ou seja, aqueles sobre os quais o paciente e mesmo a equipe de saúde podem atuar, são dislipidemias, Hipertensão Arterial (HAS), tabagismo, Diabetes Mellitus (DM), sedentarismo, estresse e obesidade. Outros FR podem também estar

\footnotetext{
* Dissertação de Mestrado apresentada à Escola de Enfermagem de Ribeirão Preto da Universidade de São Paulo (apresentação condensada)

** Professor Colaborador do Departamento de Enfermagem da Faculdade de Ciências Médicas - UNICAMP

*** Professor Doutor do Departamento de Enfermagem Geral e Especializada da Escola de Enfermagem de Ribeirão Preto - USP
} 
associados ao desenvolvimento da DAC como a ingesta excessiva de bebidas alcoólicas, menopausa, uso de contraceptivos orais, hiperuricemia, taxa de fibrinogênio aumentada e outros, os quais necessitam, porém, de maiores estudos para sua elucidação (BRASIL. Ministério da Saúde, 1993a)****.

GOLDMAN \& COOK (1984) estimaram que mais da metade (54\%) do declínio da taxa de mortalidade para a DAC nos EUA entre 1968 e 1978 foi relacionada à mudanças de estilo de vida, especificamente com a diminuição de níveis de colesterol sérico $(30 \%)$ e o abandono do hábito de fumar $(24 \%)$.

A alta incidência da DAC em nosso meio está, em parte, relacionada ao fato de encontrarmos em nossa população um estilo de vida que propicia o desenvolvimento dos FR que, já comprovadamente, contribuem para o aumento do número de indivíduos acometidos por essa doença. Esses FR que atuam sobre o desenvolvimento da DAC podem ou não estar presentes dependendo do estilo de vida adotado pelos indivíduos.

LALONDE (1974) define estilo de vida como um "conjunto de decisões individuais que afetam a saúde e sobre os quais se pode exercer certo grau de controle. As decisões e os hábitos pessoais que são maus para a saúde, criam riscos originados pelo próprio indivíduo. Quando estes riscos resultam em enfermidade ou morte se pode afirmar que o estilo de vida contribuiu ou causou a enfermidade ou disfunção". LALONDE (1974), cujo modelo de campo de saúde foi utilizado como referencial teórico deste estudo, propõe uma estrutura mais abrangente envolvendo entre os seus componentes dados referentes não só à biologia humana, como aqueles relacionados ao meio ambiente, à organização da atenção à saúde e dados referentes ao estilo de vida.

No estudo Lifestyle Heart Trial, ORNISH et al. (1990) demonstraram que mudanças no estilo de vida (dieta vegetariana, abandono do fumo, atividade física regular e manejo do estresse) foram associadas à regressão de lesões ateroscleróticas, analisadas angiograficamente após um ano da adoção do estilo de vida proposto.

Parece-nos provável que a modificação dos comportamentos não saudáveis presentes no estilo de vida pode excluir ou controlar a intensidade dos FR, o que por sua vez, parece diminuir a ocorrência, prevenir recorrências e melhorar o prognóstico de indivíduos que sofreram IAM. A magnitude do problema levou-nos a refletir sobre a necessidade de investir-se na educação do indivíduo que já apresentou uma manifestação grave da DAC, como o IAM, a fim de contribuir para a sua qualidade de vida e prevenir a reincidência de infarto.

Porém, como atingir este objetivo? Sempre foi e ainda constitui um desafio para os profissionais de saúde o desenvolvimento de estratégias educacionais que realmente possam auxiliar pacientes coronariopatas a se desvencilharem de hábitos tão marcantes. Tais hábitos podem estar tão enraizados no estilo de vida dessas pessoas, que somente a informação recebida através de planos de prevenção, realizados de uma maneira mais ampla e inespecífica, não tem sido suficiente para alterar comportamentos prejudiciais à saúde de uma forma efetiva e duradoura.

Por outro lado, o modo como são concebidos e implantados esses programas parece ser determinante importante do seu sucesso. As extrapolações de experiências na elaboração de programas de prevenção parecem não ser adequadas, principalmente quando feitas a partir de países desenvolvidos para países onde predomina uma população de classe sócio-econômica menos favorecida. $\mathrm{O}$ conhecimento da população a qual se destinam tais programas é fundamental para a determinação de intervenções que sejam efetivas na promoção de modificações de comportamento necessárias para a adoção de um estilo de vida saudável.

Preocupados com a reabilitação dos pacientes infartados, atendidos no serviço de Cardiologia do Hospital de Clínicas da Universidade Estadual de Campinas (HC-UNICAMP), detectamos a necessidade de conhecer melhor essas pessoas, a fim de podermos propor um programa educacional que atenda a sua realidade. Neste sentido, tivemos como objetivo deste estudo:

1) caracterizar o perfil dos pacientes que tenham sofrido o primeiro episódio de IAM e que foram atendidos na Unidade Coronária do HC-UNICAMP; 2) identificar a presença de FR para IAM, com destaque àqueles modificáveis pela equipe de saúde e pelo próprio paciente.

\section{METODOLOGIA}

O presente estudo foi realizado na Unidade Coronária, integrante do Núcleo de Assistência Médica Intensiva do HC-UNICAMP. A população foi constituída por 78 pacientes com diagnóstico de

\footnotetext{
* A partir desta, será citado: BRASIL. MS, 1993
} 
IAM, primeiro episódio, que estiveram internados em tal unidade no período de maio a dezembro de 1994. Foram excluídos aqueles pacientes que não concordaram em participar do estudo e aqueles que não possuíam capacidade de comunicar-se verbalmente.

Os dados foram obtidos através de entrevista semi-estruturada com o paciente e consulta ao prontuário hospitalar para levantamento de alguns dados de interesse. Para realização da entrevista foi elaborado um roteiro (Anexo 1), o qual seguiu os pressupostos do modelo de campo de saúde proposto por LALONDE (1974).

Ressaltamos, que pelo fato deste trabalho fazer parte de um estudo mais abrangente serão apresentados neste momento, somente alguns dos resultados obtidos com a aplicação do Anexo 1, os demais dados deverão ser divulgados em trabalhos subseqüentes. Os resultados encontrados foram expressos em números absolutos e índices percentuais.

\section{RESULTADOS E DISCUSSÃO}

Procedendo à caracterização do grupo estudado, encontramos uma maior incidência de IAM nos homens $(56,4 \%)$, de cor branca $(82,0 \%)$, casados $(65,4 \%)$, analfabetos ou com primeiro grau incompleto $(84,6 \%)$ e com renda inferior a três salários mínimos $(53,9 \%)$. Quanto à ocupação 47,5\% dos pacientes eram ativos e 48,6\% desenvolviam atividades classificadas no grupo 7/8/9 da Classificação Brasileira de Ocupações (BRASIL. Ministério do Trabalho, 1994), destacando-se as ocupações de pedreiro e operários de fábrica. Eram provenientes da cidade de Campinas e região 82,0\% dos pacientes, sendo que $80,7 \%$ residia na zona urbana.

Ao compararmos a distribuição dos pacientes infartados por faixa etária em relação ao sexo, podemos observar, como mostra o Gráfico 1, que a maior incidência de pacientes infartados do sexo masculino ocorreu na faixa etária dos 40 aos 70 anos (35,9\%); já a do sexo feminino entre 60 e 80 anos (29,6\%). A partir dos 80 anos de idade, observamos que esta incidência se igualou. Nossos dados, apesar de se referirem a um grupo hospitalar restrito, também evidenciaram, a exemplo dos estudos de Framingham, que no sexo masculino essa doença se manifestou aproximadamente 10 anos antes que no sexo feminino (KANNEL et al., 1961).

No que se refere aos dados relacionados ao Atendimento de Saúde, verificamos que todos os pacientes referiram a necessidade de assistência à saúde, sendo os serviços de atenção primária o mais utilizado pelos pacientes $(37,2 \%)$. O principal motivo da procura dos serviços da saúde foi o controle periódico de determinadas doenças como HAS, DM entre outras realizado por $48,7 \%$ dos pacientes.

GRÁFICO 1. Distribuição dos pacientes com primeiro episódio de IAM, segundo a idade e o sexo

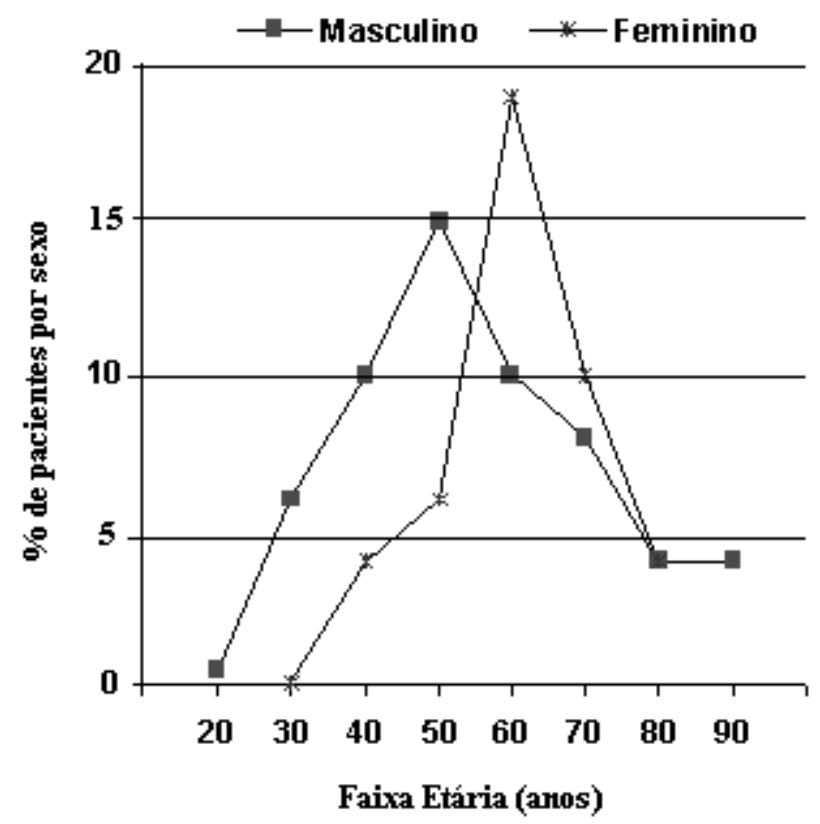

Ao analisarmos o conhecimento dos pacientes em relação ao seu diagnóstico verificamos que 53,9\% (42) mostraram ter algum grau de informação sobre a sua doença. Destes, $47,7 \%$ (20) referiram somente o nome da doença, 33,3\% (14) relataram dados da fisiopatologia como "veia entupida do coração" e 19,0\% (8) referiram nome da doença e dados da fisiopatologia simultaneamente. Dos 36 pacientes que referiram não conhecer o seu diagnóstico, a maioria $(63,9 \%)$ não sabia o motivo da internação e os outros relacionaram a sintomatologia apresentada a outras patologias como "doença do pulmão", "gastrite", "derrame" entre outras.

Procurando obter dados sobre o conhecimento de 72 pacientes infartados sobre sua doença, DUARTE (1978) observou que $36,0 \%$ dos pacientes referiram não ter nenhum conhecimento sobre sua doença. Apenas $9,7 \%$ emitiram respostas que revelavam algum conhecimento tais como "necrose de uma área do coração" e "obstrução de coronária”. 
Ao indagarmos os pacientes a respeito do que eles gostariam de saber sobre a sua doença constatamos que $55,1 \%$ (43) desejavam obter informações sobre a sua doença. Destes pacientes, $32,5 \%$ (14) queriam informações sobre o seu diagnóstico; 18,6\% (8) mostravam-se preocupados com os meios de prevenção relacionados à sua doença, $16,3 \%$ (7) gostariam de informações referentes as causas do IAM, $14,1 \%$ (6) sabiam o seu diagnóstico, porém não compreendiam o seu significado, 9,3\% (4) sentiram a necessidade de ser orientados em relação à reabilitação, 4,6\% (2) tinham dúvidas quanto ao tratamento e outros $4,6 \%$ (2) desejavam informações quanto à probabilidade de reinfarto.

No que se refere ao Estilo de vida, ao questionarmos os pacientes quanto à associação entre os FR e a ocorrência de IAM, observamos uma porcentagem significativa $(60,0 \%)$ de indivíduos que relacionaram o infarto a algum hábito do seu dia-adia. Entre as causas de IAM apontadas pelos pacientes, o estresse referido como "tensão nervosa" foi o mais citado (Gráfico 2).

GRÁFICO 2. Freqüência das causas apontadas pelos 78 pacientes como responsável pela ocorrência de IAM*

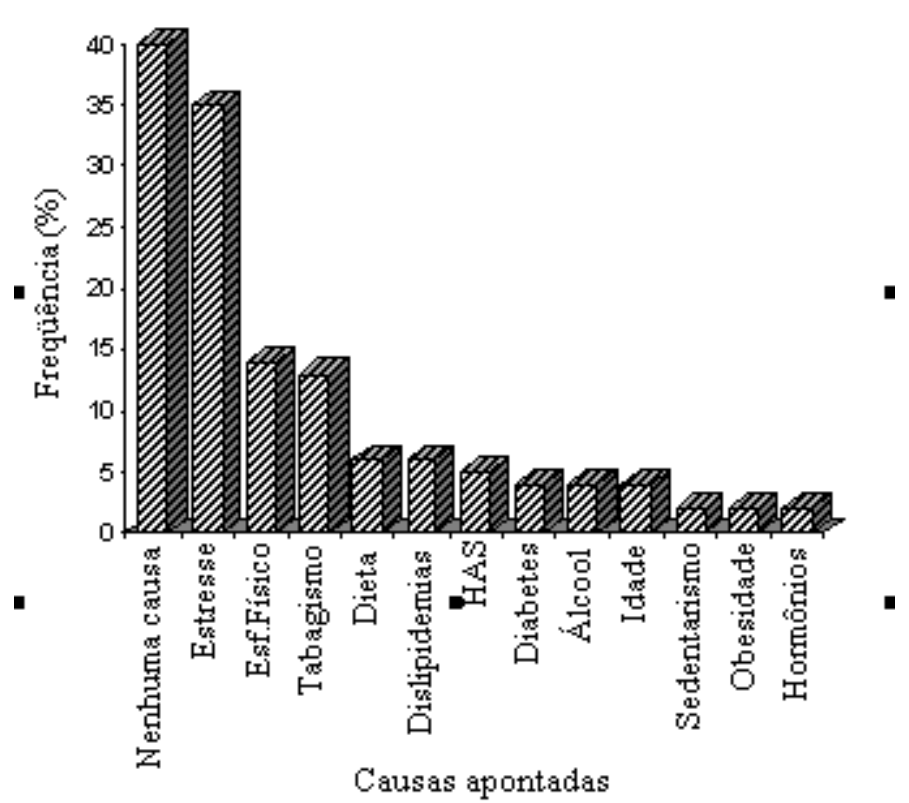

* Houve pacientes que apontaram mais de uma causa

Observamos que os dois motivos mais citados - o estresse e o esforço físico - são aqueles que estão presentes no seu dia-a-dia, quer no ambiente de trabalho ou doméstico. Enfatizamos, mais uma vez, a necessidade de levantar tais subsídios para a elaboração de um programa de orientação, principalmente se considerarmos que um dos fatores mais citados como causa de IAM, que foi o esforço físico, não tem sido definido como FR para a DAC.
Os dados referentes à alimentação, neste estudo, foram levantados com o objetivo de determinar as peculiaridades do hábito alimentar do grupo estudado e de identificar a presença ou não de certos alimentos sabidamente relacionados ao desenvolvimento da DAC. Para avaliarmos o grupo estudado em relação à ingestão de uma dieta equilibrada, optamos por agrupar os alimentos consumidos por estes pacientes em três grandes grupos: alimentos construtores, energéticos e reguladores, compostos pelos seus subgrupos conforme Anexo 2.

Os critérios de avaliação dietética utilizados para verificar se a dieta era ou não equilibrada foram o consumo de pelo menos um alimento de cada grupo em cada refeição e o consumo de pelo menos um alimento de cada subgrupo no total do dia.

Aproximadamente sessenta e cinco por cento (51) dos pacientes faziam uma refeição considerada equilibrada no almoço. Dos 34,6\% (27) pacientes que não possuíam uma dieta equilibrada nessa refeição, o componente ausente pertencia ao grupo dos alimentos reguladores. Verificamos que ocorre, no jantar, uma inversão em relação ao almoço quando $61,4 \%$ (48) dos pacientes não apresentavam uma dieta equilibrada, com predomínio de alimentos energéticos (arroz, batata, entre outros) (Gráfico 3).

GRÁFICO 3. Distribuição dos pacientes segundo o consumo no almoço e no jantar de pelo menos um componente de cada grupo de alimentos

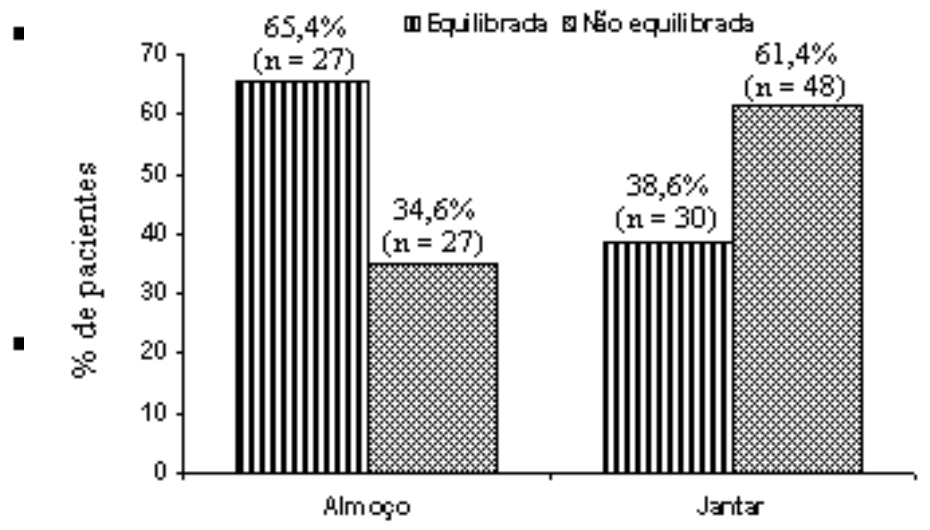

No grupo de alimentos reguladores, destacase a importância da ingestão das fibras solúveis como a pectina que estão associadas a diminuição dos níveis de colesterol total. Estão presentes na maçã e frutas cítricas que devem fazer parte de uma dieta equilibrada (STOY, 1990). As fibras insolúveis, cujo componente fundamental é a celulose, como por exemplo as verduras, não tem ação sobre o colesterol, mas são úteis na diminuição da ingestão calórica (FORTI, 1989; STOY, 1990). 
Ao coletarmos os dados referentes ao consumo de alimentos construtores, nossa preocupação foi obter informações sobre um grupo de alimentos que pode ser considerado de risco por estar relacionado ao desenvolvimento de doenças crônico-degenerativas principalmente pelo seu alto teor de colesterol. Está extensamente registrado na literatura mundial, que para evitar o aumento dos níveis séricos de colesterol deve-se restringir a ingestão de determinados alimentos, entre eles a gema de ovo, carne vermelha, carne suína, vísceras, pele de animais (peru, frango) alguns animais marinhos (camarão, lagosta, sardinha, frutos do mar). As partes branca das aves e os peixes são os que contém menor quantidade de colesterol (STOY, 1990; HADLEY \& SAAMANN, 1991; SOCIEDADE BRASILEIRA DE CARDIOLOGIA, 1993*****).

Deste modo, ao analisarmos o consumo dos alimentos construtores pelo grupo estudado, verificamos que estes eram ingeridos pela maioria dos pacientes, porém com uma freqüência muito pequena, ou seja, raramente ou menos que três vezes por semana. A baixa freqüência no consumo desses alimentos parece-nos compatível com o nível sócioeconômico do grupo em questão (Tabela 1).

Verificamos que todos os pacientes referiram o consumo de alimentos energéticos, sendo que $83,3 \%$ (65) utilizavam gordura vegetal com predomínio do óleo de soja (Tabela 2). Todos os pacientes utilizavamse de frituras como modo de preparo de seus alimentos. Destaca-se que 52,6\% (41) dos pacientes o fazia diariamente ou mais que três vezes por semana.

Além do consumo de colesterol, o elevado consumo de gorduras saturadas também é associado ao desenvolvimento de DAC, enquanto o consumo de gorduras insaturadas parece estar associado à diminuição deste risco (HADLEY \& SAAMANN, 1991). Os alimentos ricos em gorduras saturadas e que, portanto, também devem ser restritos, são as gorduras de origem animal (gordura das carnes, leite e derivados), alguns óleos vegetais (palma, coco e dendê) e alguns frutos principalmente o abacate e o coco. (FORTI, 1989; HADLEY \& SAAMANN, 1991; SBC, 1993).

TABELA 1. Distribuição dos pacientes com primeiro episódio de IAM, segundo a frequiência do consumo de alimentos construtores

\begin{tabular}{|c|c|c|c|c|c|c|c|c|c|c|c|c|c|c|}
\hline \multirow{4}{*}{$\begin{array}{l}\text { FREQÜÊNCLA DO } \\
\text { CONSUMO DE } \\
\text { ALIMENTOS } \\
\text { CONSTRUTORES }\end{array}$} & \multicolumn{14}{|c|}{ ALIMENTOS CONSTRUTORES } \\
\hline & \multicolumn{14}{|c|}{ TIPOS } \\
\hline & \multicolumn{2}{|c|}{ ovo } & \multicolumn{2}{|c|}{ LEITE } & \multicolumn{2}{|c|}{ QUEIJOS } & \multicolumn{2}{|c|}{$\begin{array}{l}\text { CARNE } \\
\text { BOVINA }\end{array}$} & \multicolumn{2}{|c|}{$\begin{array}{l}\text { CARNE } \\
\text { SUIINA }\end{array}$} & \multicolumn{2}{|c|}{ AVES } & \multicolumn{2}{|c|}{ PEIXES } \\
\hline & n & $(\%)$ & n & $(\%)$ & n & $(\%)$ & n & $(\%)$ & n & $(\%)$ & n & $(\%)$ & n & $(\%)$ \\
\hline diariamente & 2 & 2,6 & 47 & 60,2 & -- & - & 13 & 16,7 & 2 & 2,6 & 5 & 6,5 & -.- & -- \\
\hline $\begin{array}{l}\text { mais que } 3 \text { vezes } \\
\text { por semana }\end{array}$ & 12 & 15,4 & 5 & 6,5 & -- & -- & 25 & 32,1 & 4 & 5,1 & 26 & 33,3 & -- & -- \\
\hline $\begin{array}{l}\text { menos que } 3 \text { vezes } \\
\text { por semana }\end{array}$ & 28 & 35,9 & 7 & 8,9 & -- & -- & 26 & 33,3 & 13 & 16,7 & 28 & 35,8 & -- & -- \\
\hline raramente & 28 & 35,9 & 9 & 11,5 & 62 & 79,5 & 10 & 12,8 & 29 & 37,1 & 13 & 1677 & 16 & 20,5 \\
\hline não consome & 8 & 10,2 & 10 & 12,8 & 16 & 20,5 & 4 & 5,1 & 30 & 38,5 & 6 & 7,7 & 62 & 79,5 \\
\hline TOTAL & 78 & 100,0 & 78 & 100,0 & 78 & 100,0 & 78 & 100,0 & 78 & 100,0 & 78 & 100,0 & 78 & 100,0 \\
\hline
\end{tabular}

TABELA 2. Distribuição dos pacientes com primeiro episódio de IAM, segundo o tipo de gordura utilizada no preparo de alimentos

\begin{tabular}{lcc}
\hline TIPO DE GORDURA & n & (\%) \\
\hline Origem animal & 13 & 16,7 \\
- banha de porco* & & 62,8 \\
Origem vegetal & 49 & 5,1 \\
- soja & 4 & 1,3 \\
- milho & 1 & 14,1 \\
- azeite de oliva & 11 & 78 \\
- não soube referir** & 700,0 \\
\hline TOTAL & 78 \\
\hline
\end{tabular}

* todos os pacientes que referiram uso de banha de porco, faziam-no em associação ao óleo de soja

** neste subgrupo, os pacientes referiram consumo de óleo vegetal, porém sem saber especificar o tipo 
No grupo estudado, verificamos que houve um nítido predomínio da utilização de gorduras vegetais sobre as animais destacando-se o consumo de óleo de soja, o que constitui em uma medida de menor risco em relação à DAC. Porém, o uso de frituras é elevado aumentando assim o consumo de gorduras mesmo que insaturadas.

Acreditamos que esses dados referentes à alimentação, possam fornecer substrato para nortear a atuação dos profissionais de saúde na educação dos indivíduos quanto à importância da adoção de uma dieta equilibrada e quanto à necessidade de limitar o consumo de determinados alimentos que em excesso possam favorecer o desenvolvimento e/ou progressão da DAC.

Em relação ao hábito de fumar, encontramos que 44,9\% (35) dos pacientes eram fumantes e entre os não fumantes, verificamos que $33,3 \%$ (26) dos pacientes nunca haviam fumado e $21,8 \%$ (17) eram ex-fumantes (Gráfico 4).

GRÁFICO 4. Distribuição dos pacientes com primeiro episódio de IAM, segundo o hábito de fumar

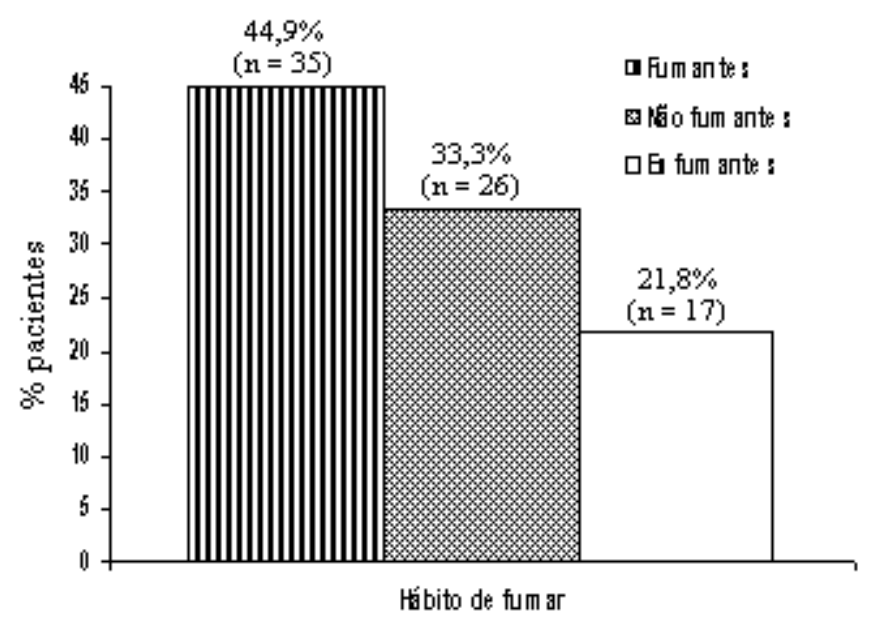

Quanto aos não fumantes, constatamos que $52,0 \%$ (22) foram considerados fumantes passivos, ou seja, inalavam constantemente a fumaça de outros fumantes, aumentando deste modo o número de indivíduos expostos ao risco do tabagismo em relação ao desenvolvimento da DAC. No MULTIPLE RISC FACTOR INTERVENTIONAL TRIAL pacientes não fumantes do sexo masculino foram estudados de acordo com a exposição à fumaça de cigarros de suas esposas fumantes. Os resultados mostraram que esses fumantes passivos tinham níveis elevados de monóxido de carbono expirado, uma diminuição da função pulmonar e aumento do risco de desenvolvimento da DAC (SVENDSEN et al., 1987). Consideramos, portanto, que no grupo estudado o tabagismo representou um importante FR para essa doença.
Ao analisarmos o FR sedentarismo, constatamos, como mostra a Tabela 3 que 76,9\% (60) dos pacientes não exerciam nenhuma atividade física. Da pequena parcela que realizava alguma atividade física, 19,2\% (15) eram homens e 3,8\% (3) mulheres.

Tabela 3. Distribuição dos pacientes com primeiro episódio de IAM, segundo a realização de atividade física e o sexo

\begin{tabular}{|c|c|c|c|c|c|c|}
\hline \multirow{3}{*}{$\begin{array}{l}\text { ATIVIDADE } \\
\text { FISICA }\end{array}$} & \multicolumn{6}{|c|}{ SEXO } \\
\hline & \multicolumn{2}{|c|}{ MASCULINO } & \multicolumn{2}{|c|}{ FEMIIIINO } & \multicolumn{2}{|c|}{ TOTAL } \\
\hline & $\mathbf{n}$ & $(\%)$ & $\mathbf{n}$ & $(\%)$ & $\mathbf{n}$ & $(\%)$ \\
\hline $\operatorname{sim}$ & 15 & 19,2 & 3 & 3,8 & 18 & 23,1 \\
\hline กล๊o & 28 & 35,9 & 32 & 41,0 & 60 & 76,9 \\
\hline TOTAL & 43 & 55,1 & 35 & 44,9 & 78 & 100,0 \\
\hline
\end{tabular}

Estudos, como o de EKELUND et al. (1988) evidenciam que a atividade física regular diminui o risco de distúrbios ateroscleróticos. Neste estudo consideramos como atividade física regular aquela recomendada pela INTERNATIONAL LIPID INFORMATION BUREAU (ILIB) LATINO AMÉRICA (1994), ou seja, 20 a 30 minutos de atividade aeróbica 3 a 5 vezes por semana para alcançar 60 a $90 \%$ de condicionamento vascular ótimo. De acordo com esta recomendação, do total de pacientes estudados, apenas 14,1\% (11) exerciam uma atividade física que pudesse ser considerada protetora em relação à DAC.

Em relação à Biologia do indivíduo, conforme o Modelo de Campo de Saúde proposto por LALONDE (1974), realizamos o cálculo de Índice de Massa Corporal (IMC) para detectar a presença do FR obesidade no grupo estudado. Para tanto, utilizamos a classificação da INTERNATIONAL LIPID INFORMATION BUREAU (ILIB) LATINO AMÉRICA (1994), que utiliza o IMC para determinar os diferentes graus de obesidade que variam de leve a severa (I, II, III). Encontramos em aproximadamente $40 \%$ dos pacientes a obesidade grau I e II (Tabela 4).

Estudos têm sugerido que, além da obesidade, os padrões de distribuição da gordura corporal podem ter efeitos importantes no risco de DAC. A deposição da gordura na região abdominal tem sido associada ao aumento do risco de distúrbios coronários tanto em homens como em mulheres. Segundo PEIRIS et al.(1989) esta obesidade designada "obesidade centrada no abdome", parece ter maior valor preditivo de morbidade e mortalidade do que a quantidade de gordura total. No entanto FARMER \& GOTTO JUNIOR (1992) consideram que o IMC tem sido o melhor índice para estimar o excesso de peso. 
No Brasil, aproximadamente 27 milhões de adultos brasileiros apresentam algum grau de excesso de peso, ou seja, um IMC superior à $25 \mathrm{Kg} / \mathrm{m}^{2}$. Destes, aproximadamente 11 milhões são homens e 16 milhões são mulheres (BRASIL. MS, 1993a).

Embora existam estudos mostrando que a perda de peso acarreta a redução de outros FR que freqüentemente acompanham a obesidade, CUNNINGHAM (1992) destaca que nenhum estudo de maior abrangência foi realizado para estudar a perda de peso como forma de reduzir mortalidade para DAC.

TABELA 4. Distribuição dos pacientes com primeiro episódio de IAM, segundo IMC e o sexo

\begin{tabular}{|c|c|c|c|c|c|c|}
\hline \multirow{3}{*}{$\underset{\mathrm{Kg} / \mathrm{m}^{2}}{\mathrm{IMC}}$} & \multicolumn{6}{|c|}{ SEXO } \\
\hline & \multicolumn{2}{|c|}{ MASCULINO } & \multicolumn{2}{|c|}{ FEMININO } & \multicolumn{2}{|c|}{ TOTAL } \\
\hline & $\mathbf{n}$ & $(\%)$ & $\mathbf{n}$ & $(\%)$ & $\mathbf{n}$ & $(\%)$ \\
\hline Normal $(<25)$ & 21 & 27,0 & 15 & 19,2 & 36 & 46,1 \\
\hline Sobrepeso $(25-27,5)$ & 5 & 6,4 & 6 & 7,7 & 11 & 14,1 \\
\hline Obesidade grau I ou leve $(27,5-29,9)$ & 15 & 19,2 & 4 & 5,1 & 19 & 24,3 \\
\hline Obesidade grau II ou moderada $(30-39,9)$ & 3 & 3,8 & 9 & 11,5 & 12 & 15,4 \\
\hline Obesidade grau III ou severa $(>40)$ & -- & -- & -- & -- & -- & -- \\
\hline TOTAL & 44 & 56,4 & 34 & 43,6 & 78 & 100,0 \\
\hline
\end{tabular}

No que se refere aos antecedentes familiares, verificamos a sua existência em relação à HAS $(50 \%)$, IAM $(34,6 \%)$, morte súbita $(32,0 \%)$, DM $(30,8 \%)$, Acidente Vascular Cerebral (AVC) $(29,5 \%)$ e dislipidemias $(11,5 \%)$.

No que se refere aos antecedentes pessoais de risco, constatamos que 69,2\%(54) dos pacientes eram hipertensos, 44,9\%(35) eram tabagistas, $29,5 \%(23)$ eram diabéticos 6,4\%(5) apresentavam AVC e 6,4\%(5) possuíam antecedente para Doença Vascular Periférica (DVP) (Gráfico 5).

No Brasil, estimou-se que doze milhões de brasileiros com mais de 20 anos de idade o que corresponde a $15 \%$ da população desta faixa etária, são hipertensos (BRASIL. MS, 1993a). Em relação ao DM, a média geral de prevalência encontrada em nove capitais brasileiras foi de $7,6 \%$, o que é pouco superior à encontrada na população dos EUA, que corresponde a 6,6\% (BRASIL. MS,1993b).

Com o objetivo de levantar o perfil lipídico dos pacientes estudados, foram dosados nas primeiras $48 \mathrm{~h}$ de IAM os níveis séricos de colesterol total. Devido ao fato do IAM poder alterar as concentrações de lipídios no plasma, é freqüentemente recomendado que a dosagem dessas variáveis sejam feitas somente três meses após ter ocorrido o IAM. Alguns estudos indicam que o nível de colesterol total diminui na fase aguda do IAM e que por este motivo não poderia refletir o nível basal de lipídios séricos (FYFE et al., 1971; ALPERT \& BRAUNWALD,1980). Entretanto GORE et al. (1984), analisando uma coorte de indivíduos arrolados no estudo de Framingham, observaram que não houve diferença estatisticamente significativa entre os valores de lipídios séricos dosados até 24 horas após o episódio de IAM e após 2 anos de alta hospitalar. Portanto, para estes autores os níveis de colesterol medido até 24 horas de IAM "refletem acuradamente o nível de base e podem ser utilizados em programas de intervenção".

Gráfico 5. Prevalência dos antecedentes pessoais de HAS, Tabagismo, DM, AVC e DVP, entre os pacientes com primeiro episódio de IAM

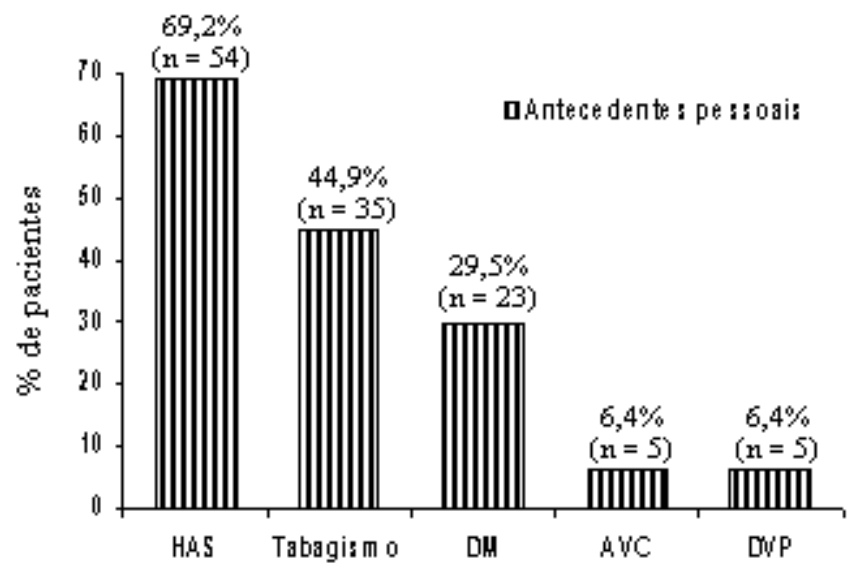

* Considerou-se o total da freqüência de cada fator de risco (isolado ou associado a outro fator de risco)

Foi possível realizar a dosagem do colesterol total de 55 pacientes; os demais (23) ou foram admitidos após $48 \mathrm{~h}$ de IAM ou apresentaram problemas técnicos na realização das dosagens, o que inviabilizou sua inclusão na avaliação desta variável. Utilizamos como valores de referência para o 
colesterol total aquele recomendado pela GEPA, $\mathrm{SBC}^{+}, \mathrm{SBPC}^{\sharp}$ e ASBAC ${ }^{++}$o qual é baseado no Consenso do Programa Nacional do Colesterol nos EUA (National Cholesterol Education Program NCEP).

Deste modo, ao analisarmos a distribuição dos pacientes em relação ao nível sérico de colesterol total dosado até as 48horas de IAM, encontramos que, dentre os 55 pacientes analisados, $29(52,7 \%)$ apresentavam nível sérico de colesterol total desejável $(<200 \mathrm{mg} / \mathrm{dl})$. Para os 26 pacientes $(47,3 \%)$ que apresentavam nível sérico de colesterol total limítrofe ou acima do desejável, tivemos que 13 pacientes $(50,0 \%)$ eram do sexo feminino e $13(50,0 \%)$ do sexo masculino (Tabela 5).

TABELA 5. Distribuição dos 55 pacientes com primeiro episódio de IAM, segundo o nível sérico de colesterol total dosado até 48 horas de IAM e o sexo

\begin{tabular}{|c|c|c|c|c|c|c|}
\hline \multirow{3}{*}{$\begin{array}{c}\text { COLESTEROL } \\
\text { TOTAL } \\
\mathrm{mg} / \mathrm{dl}\end{array}$} & \multicolumn{6}{|c|}{ SEXO } \\
\hline & \multicolumn{2}{|c|}{ MASCULINO } & \multicolumn{2}{|c|}{ FEMININO } & \multicolumn{2}{|c|}{ TOTAL } \\
\hline & n & $(\%)$ & n & $(\%)$ & n & $(\%)$ \\
\hline$<200$ (desejável) & 21 & 38,1 & 8 & 14,5 & 29 & 52,7 \\
\hline 201 - 239 (limitrofe) & 9 & 16,3 & 5 & 9,0 & 14 & 25,4 \\
\hline$>240$ (elevado) & 4 & 7,2 & 8 & 14,5 & 12 & 21,8 \\
\hline TOTAL & 34 & 61,6 & 21 & 38,0 & 55 & 100,0 \\
\hline
\end{tabular}

Segundo STOVSKY (1992), a NCPE recomenda que todos os indivíduos com níveis desejáveis de colesterol total devem permanecer com dieta geral, porém deverão receber informações sobre os FR para a DAC e ser reavaliados a cada cinco anos. Indivíduos que possuem níveis limítrofes de colesterol total, devem ter este valor confirmado por novos testes. Se este valor for confirmado e se o paciente não possuir DAC e não tiver no mínimo dois FR para esta doença, ele deve receber somente orientação e reavaliação em um ano. Por outro lado, se estes pacientes possuírem DAC ou tiverem dois outros FR, então devem ser considerados de alto risco. Portanto todos os pacientes deste estudo que tiveram seu colesterol total acima de $200 \mathrm{mg} / \mathrm{dl}$, isto é, acima do nível desejável, foram considerados de alto risco.

$$
\text { Considerando-se os achados do }
$$
SCANDINAVIAN SIMVASTATIN SURVIVAL STUDY GROUP (1994), onde se constatou o aumento da sobrevida dos indivíduos coronariopatas que tiveram diminuição do colesterol sérico, ressaltase mais uma vez a importância do controle desse fator de risco.

Com relação à associação dos FR modificáveis para a DAC, constatamos que, do total de pacientes, apenas $2,6 \%$ (2) não apresentavam nenhum FR, $12,8 \%$ (10) possuíam 1 fator e 61,5\% (48) possuíam três fatores ou mais ( Gráfico 6).

\footnotetext{
GEPA - Grupo de Estudo e Pesquisa em Aterosclerose

${ }^{+}$SBC - Sociedade Brasileira de Cardiologia

${ }^{¥}$ SBPC - Sociedade Brasileira de Patologia Clínica

${ }^{++}$ASBAC - Associação Brasileira de Análises Clínicas
}

Segundo inúmeros estudos, quanto maior o número de $\mathrm{FR}$, maior é o risco de desenvolvimento de DAC (BANCO MUNDIAL, 1991; CUNNINGHAM, 1992; BRASIL. MS, 1993a; KANNEL, 1995).

GRÁFICO 6. Distribuição dos pacientes com primeiro episódio de IAM, segundo a associação dos fatores de risco modificáveis para a DAC (tabagismo, dislipidemias, HAS, DM, sedentarismo e obesidade)

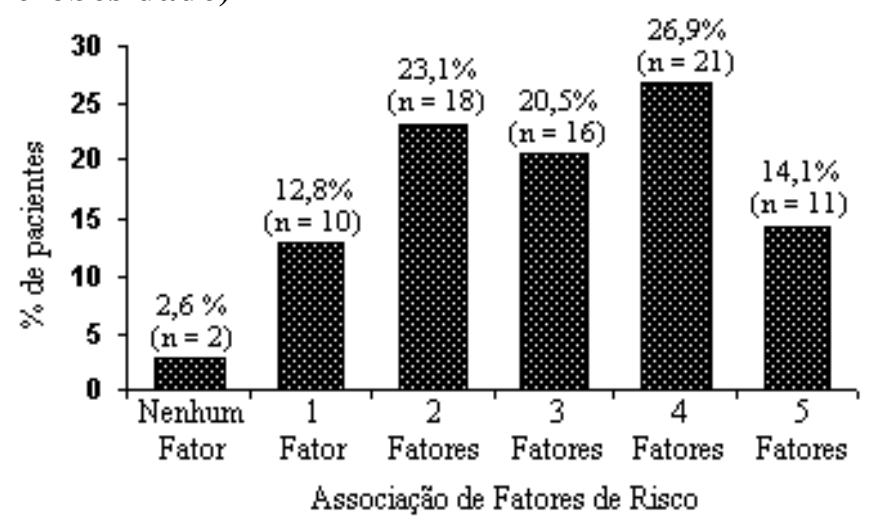

\section{CONSIDERAÇÕES FINAIS}

Os resultados deste estudo apontam para o fato de estarmos trabalhando com pessoas que possuem, em seu estilo de vida, hábitos que propiciam a manutenção ou progressão da DAC e suas diversas formas de manifestação clínica. Constatamos no 
grupo estudado uma alta prevalência de FR tais como HAS, tabagismo, sedentarismo, obesidade entre outros, porém a maioria dos pacientes, apesar de apresentar três ou mais FR para a DAC, não correlacionou esses fatores com o infarto que os acometeu, o que vem confirmar o seu baixo nível de informação e a necessidade de um programa educacional específico para esse grupo. Com o empenho de uma equipe multiprofissional, talvez se possam desenvolver mecanismos que levem os indivíduos a assumirem uma atitude ativa diante de sua doença, conhecendo e controlando os FR presentes no seu estilo de vida. De qualquer forma, a educação em saúde só poderá ser um instrumento de trabalho para os profissionais de saúde, se conhecerem a população que estão assistindo para, dessa forma, poderem levar em consideração suas peculiaridades, suas limitações e consequentemente as reais possibilidades de atuação da equipe multiprofissional.

\section{LIFE STYLE AND RISK FACTORS OF PATIENTS WITH A FIRST EPISODE OF ACUTE MYOCARDIAL INFARCTION}

The prevention of acute myocardial infarction (AMI) has been related to the identification and control of risk factors $(R F)$ present in the life style of the individuals. The aim of this study was to know the profile of patients with a first episode of AMI and to identify $R F$ for this disease. Seventy eight in patients from the Coronary Care Unity of a University Hospital were interviewed. The results showed that the life style of the studied group includes habits that collaborate to coronary artery disease maintenance or progress. These results will help to elaborate an educational program aiming to prevent reinfarction and to promote health.

KEY WORDS: myocardial infarction, life style, coronary disease

\section{ESTILO DE VIDA Y FACTORES DE RIESGO EN PACIENTES CON PRIMER EPISODIO DE INFARTO AGUDO DE MIOCARDIO}

La prevención del infarto agudo de miocardio (IAM) está relacionado con la identificación y control de los factores de riesgo (FR) presentes en el estilo de vida de estas personas. El estudio tiene por objetivo conocer el perfil de pacientes con un primer episódio de IAM, identificando los factores de riesgo para esta enfermedad. Fueron estudiados 78 pacientes atendidos en un Hospital Universitário. Los resultados mostraron que el grupo estudiado posee en su estilo de vida, hábitos que originan la mantención y/o evolución de coronariopatías. Estos datos serán base para elaborar un programa educacional teniendo como objeto la prevención del reinfarto y la promoción de la salud.

\section{REFERÊNCIAS BIBLIOGRÁFICAS}

01. ALPERT, J.S.; BRAUNWALD, E. Pathological and clinical manifestations of acute myocardial infarction. In: BRAUNWALD, E. Heart disease: a textbook of cardiovascular medicine. 3 ed. Philadelphia: WB. Saunders, 1980. cap. 20, p.1304-52.

02. BANCO MUNDIAL. Brasil novo desafio à saúde do adulto. Washington, 1991. 134 p.

03. BATLOUNI, M. Interação placa, endotélio, coagulação e isquemia aguda. RSCESP, v.3, n.2, p. 6-13, 1993.

04. BRASIL. Ministério da Saúde. Secretaria de Assistência à Saúde. Departamento de Programas de Saúde. Coordenação de Doenças Cardiovasculares. Doenças cardiovasculares no Brasil. Sistema Único de Saúde-SUS: dados epidemiológicos, assistência médica. Brasília, MS, 1993a. 36p.
05. BRASIL. Ministério da Saúde. Secretaria de Assistência à Saúde. Departamento de Assistência e Promoção à Saúde. Coordenação de Doenças Crônicodegenerativas. Manual de Diabetes. 2 ed. Brasília, MS, 1993 b. cap. 3, p.17-24: Epidemiologia do diabetes mellitus.

06. BRASIL. Ministério do Trabalho. Secretaria de Políticas de Emprego e Salário. Classificação brasileira de ocupações. Brasília: Secretaria de Políticas de Emprego e Salário, 1994. 376p.

07. CUNNINGHAM, S. The epidemiologic basis of coronary disease prevention. Nurs. Clin. North. Am., v. 27, n.1, p. 153-65, 1992.

08. DUARTE, E. Contribuição ao estudo de um programa de orientação a pacientes infartados. Ribeirão Preto, 1978. 112p. Dissertação (Mestrado) - Escola de Enfermagem, Universidade de São Paulo. 
09. EKELUND, L.G. et al. Phisical fitness as a predictor of cardiovascular mortality in assymptomatic north american men. The lipids research clinics mortality follow-up study. $\mathbf{N}$. Engl. J. Med., v. 329, n.21, p. 1379-84, 1988.

10. FARMER, J. A. ; GOTTO JUNIOR, A.M. Risk factors for coronary artery disease. In: BRAUNWALD, E. Heart disease: a textbook of cardiovascular medicine. 4 ed. Philadelphia: WB. Saunders, 1992. cap. 37, p. 1125-59.

11. FORTI, N. Como tratar e diagnosticar hipercolesterolemia. Rev. Bras. Med., v. 46, p. 39-45, 1989. Edição Especial.

12. FYFE, T. et al. Plasma-lipid changes after myocardial infarction. Lancet, v.1, p.9971001, 1971.

13. GOLDMAN, L. ; COOK, E.F. The decline in isquemic heart disease mortality rates. An analyses of the comparative effects of medical intervention and changes in lifestyle. Ann. Intern. Med., v. 101, n. 6, p.285-92, 1984.

14. GORE, J.M. et al. Validity of serum total cholesterol level obtained within 24 hours of acute myocardial infarction. Am. J. Cardiol., v. 54, n. 1, p. 722-5, 1984.

15. HADLEY, S.A. ; SAAMANN, L. Lipid physiology and nutritional considerations in coronary heart disease. Crit. Care Nurs., v. 11, n. 10, p. 28-39, 1991.

16. INTERNATIONAL LIPID INFORMATION BUREAU (ILIB) LATINO AMÉRICA. Recomendaciones de ILIB para el diagnóstico de las dislipidemias en Latino América. Cardiovasc. Risk Factors, v. 3, n. 1, p. 1027, 1994. Suplemento 1.

17. KANNEL, W.B. Natural history of cardiovascular risk. In: KAPLAN, N. Hypertension: mechanisms and therapy. Phiadelphia: Current medicine, 1995. cap. 5, p. 5.1 - 5.22.
18. KANNEL, W.B. et al. Factors of risk in the development of coronary heart disease - sixyear follow-up experience: the Framingham study. Ann. Intern. Med., v. 55, n.1, p. 33$50,1961$.

19. LALONDE, M. A new perspective on the health of canadians: a working document. Ottawa, April 1974.

20. MANCILHA-CARVALHO, J.J. Antecedentes da doença coronária: os fatores de risco. Arq. Bras. Cardiol., v. 58, n. 4, p. 263-7, 1992.

21. ORNISH, D. et al. Can lifestyle chances reverse coronary hearth disease? The lifestyle heart trial. Lancet, v. 336, n. 21, p. 129-33, 1990.

22. PEIRIS, A.N. et al. Adiposity, fat distribuition and cardiovascular risk. Ann. Intern. Med., v. 110, n. 11, p. 867-72, 1989.

23. SCANDINAVIAN SIMVASTATIN SURVIVAL STUDY GROUP. Randomised trial of cholesterol lowering in 4444 patients with coronary heart disease: the scandinavian survival study (4S). Lancet, v.344, p. 1383-9, 1994.

24.SOCIEDADE BRASILEIRA DE CARDIOLOGIA. Consenso brasileiro sobre dislipidemias: detecção, avaliação, tratamento. Arq. Bras. Cardiol., v. 61, p. 1-13, 1993. Suplemento 1.

25. STOY, D.B. Diet and drug therapy for hypercholesterolemia. Principles and perspectives for the occupational health setting. AAOHN J., v. 38, n. 5, p. 222-9, 1990.

26. STOVSKY, B. Nursing interventions for risk factor reduction. Nurs. Clin. North Am., v. 27, n. 1, p. 257-70, 1992.

27. SVENDSEN, K. H. et al. Effects of passive smoking in the multiple risk factor intervention trial. Am. J. Epidemiol., v. 126, n. 5, p. 78395, 1987. 
ROTEIRO PARA ENTREVISTA

\section{Identificação}

Nome:

Sexo: ( ) M

$\mathrm{HC}:$

( ) F $\quad$ Idade: anos

Cor: ( ) branca ( ) preta $\quad$ ( ) parda ( ) outros. Especifique:

\section{Dados Sociais e Ambientais}

2.1. Trabalho

- Ocupação:

- Tem mais de um emprego? ( ) não

( ) sim. Qual(is)?

Jornada de Trabalho:

- Local de trabalho:

- Meio de transporte utilizado para ir ao trabalho: ( ) a pé

Especifique:

- Tempo de locomoção ao trabalho: ( ) menor que 1 hora

- Satisfação no trabalho: ( ) sim não

2.2. Nível de Instrução
( ) analfabeto
( ) $1^{\circ}$ grau incompleto
( ) $1^{\circ}$ grau completo
( ) $2^{\circ}$ grau incompleto
( ) $2^{\circ}$ grau completo
( ) superior incompleto
( ) superior completo

2.3. Estado Civil
( ) solteiro
( ) casado
( ) viúvo
( ) divorciado
( ) outros. Especifique:

\subsection{Renda Familiar}

( ) menor que 3 salários mínimos ( ) entre 3 e 6 salários mínimos

( ) acima de 10 salários mínimos ( ) não sabe referir

2.5. Número de Filhos:

2.6. Local de Residência

- Perímetro: ( ) urbano

- Procedência (cidade): vivos:

( ) rural Número de pessoas que residem na mesma casa:

\section{Dados Relacionados ao Atendimento de Saúde}

3.1. Data do diagnóstico do IAM:

3.2. Paciente tem conhecimento do diagnóstico do infarto do miocárdio? ( ) sim ( ) não

Em caso de resposta afirmativa: ( ) refere o nome

( ) refere dados da fisiopatologia

3.3. O que gostaria de saber sobre sua doença?

3.4. Além do HC-UNICAMP utiliza algum outro serviço de saúde? ( ) não ( ) sim. Especifique:

Em que ocasiões:

( ) periodicamente para prevenir doenças ( ) periodicamente, pois faz tratamento médico

( ) em casos de urgência ( ) outros. Especifique:

3.5. Atualmente faz tratamento para algum tipo de doença? ( ) não ( ) sim. Especifique:

3.6. Faz uso de algum medicamento? ( ) não ( ) sim. Especifique:
Uso regular?

( ) $\operatorname{sim}$

\section{Dados Relacionados ao Estilo de Vida}

4.1. Relaciona o estilo de vida e os fatores de risco coronários com ocorrência do IAM? ( ) não （ ) sim

4.2. Quais são as causas apontadas pelo paciente para a ocorrência do seu infarto?
( ) dieta incorreta
( ) dislipidemias
( ) hipertensão arterial
( ) tabagismo
( ) diabetes mellitus
( ) sedentarismo
( ) obesidade
( ) ingestão de álcool
( ) uso de hormônios ( ) história familiar
( ) estresse
( ) raça
( ) outros. Especifique:
( ) idade

4.3. Alimentos que mais freqüentemente consome nas refeições:

Café da manhã:

Almoço:

4.4. Possui o hábito de alimentar-se no intervalo das refeições? ( ) não ( ) sim

Quais são os alimentos que mais freqüentemente consome fora das principais refeições? 
4.5. Quais dos alimentos abaixo fazem parte da sua alimentação?
( ) ovo
( ) com gema
( ) sem gema
( ) leite: ( ) integral
( ) semi-desnatado
( ) outros. Especificar:
( ) queijo:
( ) amarelo
( ) branco
( ) manteiga
( ) margarina
( ) maionese
( ) carne bovina:
( ) carne gorda
( ) carne magra
( ) carne suína
( ) aves: ( ) com pele
( ) sem pele
Frequiência: a - diariamente c - menos de 3 vezes por semana
b - mais de 3 vezes por semana
d - raramente

4.6. Alimenta-se de frituras com que freqüência?
( ) diariamente
( ) mais de 3 vezes por semana
( ) menos de 3 vezes por semana
( ) raramente

4.7. Tipo de gordura utilizada no preparo de suas refeições: ( ) vegetal ( ) animal. Especificar:

4.8. Alimenta-se de doces e de refrigerantes com que freqüência?
( ) diariamente
( ) mais de 3 vezes por semana
( ) menos de 3 vezes por semana
( ) raramente

4.9. Faz uso de bebidas alcoólicas? ( ) não ( ) sim. Tipo: Com que freqüência?
( ) diariamente
( ) mais de 3 vezes por semana
( ) menos de 3 vezes por semana
( ) nos finais de semana
( ) raramente

4.10. Tem hábito de tomar café? ( ) não ( ) sim. Quantidade: copos/dia Com que freqüência?
( ) diariamente
( ) mais de 3 vezes por semana
( ) menos de 3 vezes por semana
( ) raramente

4.11. Com relação ao hábito de fumar:
( ) fumante
( ) Fuma há quanto tempo?
( ) ex-fumante
( ) Parou há quanto tempo?
( ) Quantidade de cigarros/dia?
( ) não fumante

4.12. Se não fumante ( ou ex-fumante), compartilha de locais fechados com fumantes em seu dia-a-dia?
( ) não
( ) $\operatorname{sim}$
Onde? ( ) casa
( ) trabalho ( ) outros. Especifique:

4.13. Realiza alguma atividade física regularmente? ( ) não ( ) sim

Atividade: ( ) andar ( ) correr ( ) jogar bola ( ) nadar ( ) outros. Especifique:

Tempo:

Frequiência:

4.14. Considera-se mais estressado(a) em situações que ocorrem em que tipo de ambiente?
( ) doméstico
( ) de trabalho
( ) outros. Especifique:

4.15. Recordação de algum fato significativo ocorrido antes do IAM que tenha trazido preocupação e tristeza?
( ) não
( ) $\operatorname{sim}$

4.16. Apresenta alterações do sono com freqüência? ( ) não

4.17. Atribui essas alterações a quais fatores?

( ) mudanças de hábito (ambiente estranho, ausência de objetos familiares, horário alterado devido a trabalho ou lazer) ( ) doenças ( ) drogas ( ) álcool ( ) estado emocional ( ) outros. Especifique:

4.18. Possui vida sexual ativa? ( ) não ( ) sim

4.19. Se mulher, houve ocorrência da menopausa? ( ) não ( ) sim. Quando?
Tipo: ( ) natural
( ) cirúrgica

4.20. Faz uso de hormônios? ( ) não ( ) sim. Há quanto tempo?

4.21. Fez uso no passado de hormônios para efeitos contraceptivos? ( ) não ( ) sim. Por quanto tempo?

4.22. Fez uso de hormônios para outros fins? ( ) não ( ) sim. Especifique:

\section{Dados Relacionados à Biologia do Indivíduo}


5.2. Na família

Pai: ( ) vivo

Mãe:

( ) falecido

Causa da morte:

Irmão(s): ( ) vivo(s) ( ) falecido(s)

Causa da morte:

5.3. Na família já houve caso de:

5.3.1. Hipertensão arterial: ( ) não sabe referir ( ) não ( ) sim

Especifique:

5.3.2. Diabetes mellitus: ( ) não sabe referir ( ) não ( ) sim

Especifique:

5.3.3. Morte súbita: ( ) não sabe referir ( ) não ( ) $\operatorname{sim}$

Especifique:

5.3.4. Dislipidemias: ( ) não sabe referir ( ) não ( ) sim

Especifique:

5.3.5. Infarto do miocárdio: ( ) não sabe referir ( ) não ( ) sim

Especifique:

5.3.6. Acidente vascular cerebral: ( ) não sabe referir ( ) não ( ) sim

Especifique:

5.4. Tem (ou teve) algumas das doenças abaixo?

5.4.1. Hipertensão arterial: ( ) não sabe referir ( ) não ( ) $\operatorname{sim}$

Se afirmativo, especifique:

Há quanto tempo?

5.4.2. Diabetes mellitus: ( ) não sabe referir ( ) não ( ) sim

Se afirmativo, especifique:

Há quanto tempo?

5.4.3. Dislipidemias: ( ) não sabe referir ( ) não ( ) sim

Se afirmativo, especifique:

Há quanto tempo?

5.4.4. Doença Vascular Periférica: ( ) não sabe referir ( ) não ( ) sim

Se afirmativo, especifique:

Há quanto tempo?

5.4.5. Acidente vascular cerebral: ( ) não sabe referir ( ) não ( ) sim

Se afirmativo, especifique:

Há quanto tempo?

5.5. Perfil Lipídico:

- Colesterol total:

HDL-colesterol:

- LDL-colesterol:

VLDL-colesterol:

- Triglicérides: 
ANEXO 2

Grupo de Alimentos*

\begin{tabular}{|c|c|c|}
\hline ALIMENTOS CONSTRUTORES & ALIMENTOS REGULADORES & ALIMENTOS ENERGÉTICOS \\
\hline $\begin{array}{l}\text { 1. Fontes de Proteinas e Ferro } \\
\text { - cames (bovina, suina, aves e } \\
\text { pescado) } \\
\text { - ovos } \\
\text { - leguminosas secas } \\
\text { (feijão, ervilha, lentilha, soja, grão- } \\
\text { de-bico e amendoim) } \\
\text { 2. Fontes de Proteinas e Cálcio } \\
\text { - leite } \\
\text { - queijos } \\
\text { - iorgute } \\
\text { - coalhada }\end{array}$ & $\begin{array}{l}\text { 1. Fontes de Vitaminas, Minerais } \\
\text { e Fibras } \\
\text { - frutas ricas em vitamina C } \\
\text { (lararia, limão, mexerica, mamão, } \\
\text { goiaba, morango, caju e manga) } \\
\text { - hortaliças folhosas ricas em } \\
\text { vitamina A e ferro } \\
\text { (agrião, acelga, almeirão, couve, } \\
\text { es carola, e spina fre, mostarda, } \\
\text { salsa, rúcula) } \\
\text { - outras hortaliças e frutas }\end{array}$ & $\begin{array}{l}\text { 1. Fontes de Hidratos de carbono } \\
\text { - cereais } \\
\text { (arroz, milho, trigo, aveia, cevada } \\
\text { e centeio) } \\
\text { - farinhas e massas } \\
\text { - feculentos } \\
\text { (batata, mandioca, cará, inhame) } \\
\text { - mel e geléias } \\
\text { 2. Fontes de Gorduras } \\
\text { - óleos vegetais } \\
\text { - manteiga } \\
\text { - margarina } \\
\text { - maionese }\end{array}$ \\
\hline
\end{tabular}

* BOOG, M.C.F.; MOTTA, D.G.; BON, A.M.X. Alimentação natural: prós \& contras. São Paulo: Ibrasa, 1985. cap. 2, p. 20-51: O que precisamos saber sobre nutrição 